\title{
Decision-making for decompressive craniectomy in traumatic brain injury aided by multimodality monitoring: illustrative case
}

\author{
Myranda B. Robinson, BS, ${ }^{1}$ Peter Shin, MD, ${ }^{1}$ Robert Alunday, MD, ${ }^{1}$ Chad Cole, MD, ${ }^{1}$ Michel T. Torbey, MD, MPH, ${ }^{2}$ and \\ Andrew P. Carlson, MD, MS-CR ${ }^{1}$
}

Departments of ${ }^{1}$ Neurosurgery and ${ }^{2}$ Neurology, University of New Mexico School of Medicine, Albuquerque, New Mexico

BACKGROUND Severe traumatic brain injury (TBI) requires individualized, physiology-based management to avoid secondary brain injury. Recent improvements in quantitative assessments of metabolism, oxygenation, and subtle examination changes may potentially allow for more targeted, rational approaches beyond simple intracranial pressure (ICP)-based management. The authors present a case in which multimodality monitoring assisted in decision-making for decompressive craniectomy.

OBSERVATIONS This patient sustained a severe TBI without mass lesion and was monitored with a multimodality approach. Although imaging did not seem grossly worrisome, ICP, pressure reactivity, brain tissue oxygenation, and pupillary response all began worsening, pushing toward decompressive craniectomy. All parameters normalized after decompression, and the patient had a satisfactory clinical outcome.

LESSONS Given recent conflicting randomized trials on the utility of decompressive craniectomy in severe TBI, precision, physiology-based approaches may offer an improved strategy to determine who is most likely to benefit from aggressive treatment. Trials are underway to test components of these strategies.

https://thejns.org/doi/abs/10.3171/CASE2197

KEYWORDS multimodality monitoring; traumatic brain injury; decompressive craniectomy; intracranial pressure; cerebral autoregulation; pupillary response

Severe traumatic brain injury (TBI) represents a significant public health problem and is characterized by complex physiology, making individualized, targeted care desirable. ${ }^{1}$ Although neurocritical care and surgical strategies seek to minimize the secondary brain injury that follows the initial injury, ${ }^{2}$ it is increasingly being recognized that the physiology of the injured brain can change between patients and even within a given patient over the course of observation. This necessitates a precision medicine approach in which therapy is directed toward the individual patient's physiology. ${ }^{3}$ Multimodality monitoring (MMM) may provide better insight into the physiological changes occurring in real time, supplementing the bedside neurological examination and allowing use of directed therapies. Ideally, MMM could provide indications of a problem before it occurs, unlike a neurological examination in which the manifestation has already occurred and may be irreversible.

Various neuromonitoring tools are used to access physiological changes, with intracranial pressure (ICP) monitoring being the most common. Increased ICP can lead to a higher rate of mortality if left untreated by impairing cerebral perfusion, resulting in secondary brain injury. ${ }^{4}$ ICP (and cerebral perfusion pressure [CPP])-directed therapy is therefore currently the cornerstone of management of severe TBI. ${ }^{5}$ When ICP becomes refractory to lower tiers of treatment, decompressive craniectomy (DC) can be used as a late-tier procedure. Two recent trials, however, arrived at complex and somewhat contradictory results regarding the efficacy of this intervention, which led to complex new recommendations. ${ }^{6}$ The authors

ABBREVIATIONS CBF = cerebral blood flow; CPP = cerebral perfusion pressure; $\mathrm{DC}=$ decompressive craniectomy; GCS = Glasgow Coma Scale; ICP = intracranial pressure; $\mathrm{MAP}=$ mean arterial pressure; $\mathrm{MMM}=$ multimodality monitoring; $\mathrm{NPi}=$ Neurological Pupil index; $\mathrm{PbO}_{2}=$ brain tissue oxygenation; $\mathrm{PRx}=$ pressure reactivity; $\mathrm{TBI}=$ traumatic brain injury. 


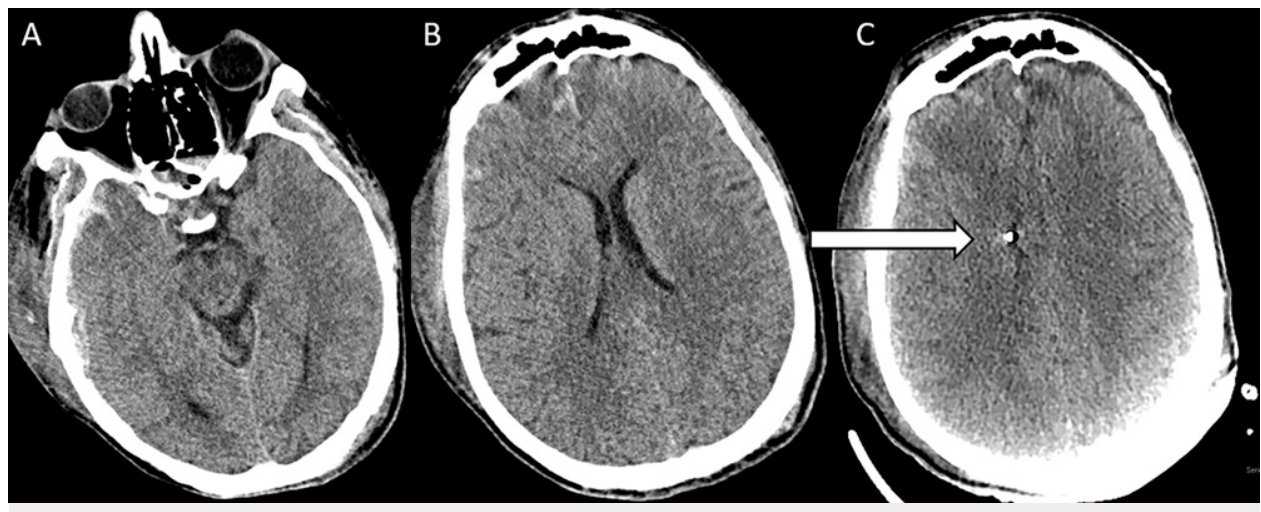

FIG. 1. Noncontrast axial CT scans of the patient's head. A and B: Initial studies with scattered contusions in the left temporal and frontal areas. There is no sulcal or basal cistern compression. C: Interval placement of the MMM bolt with external ventricular drain (arrow).

of the updated guidelines ultimately concluded that "choosing to perform a DC is not a simple decision and that the potential benefits should be balanced against the complications and likely outcomes on a case-by-case basis."

The addition of other MMM variables besides ICP may provide more insight into both baseline salvageability and the risk of further deterioration and could direct interventions. Quantitative, standardized variables such as pupillary reactivity as measured by pupillometer, ${ }^{7}$ autoregulatory measures such as pressure reactivity, ${ }^{8}$ and brain tissue oxygenation $\left(\mathrm{PbO}_{2}\right)^{9}$ may offer additional insight into impending herniation events and need for aggressive intervention. We present a case of a patient with severe TBI managed with MMM in whom these variables assisted in decision-making for DC with improvement in both monitoring variables and neurological function after intervention.

\section{Illustrative Case}

A 25-year-old male pedestrian was struck by an automobile and lost consciousness. He was brought to the University of New Mexico Emergency Department with an initial Glasgow Coma Scale (GCS) score of 4. Computed tomography of the patient's head revealed traumatic, multifocal, multicompartmental hemorrhage with mild left predominance in the middle fossa (Fig. 1). The basal cisterns remained patent without radiographic signs of severely elevated ICP.

Because of the relatively benign initial appearance, a parenchymal ICP monitor was initially placed (Hummingbird Solo, IRRAS). Initial ICP was $30 \mathrm{~mm} \mathrm{Hg}$, and the patient began to have spontaneous posturing. After standard tier 1 interventions for ICP, the patient was administered a $3 \% \mathrm{NaCl}$ bolus, which resulted in improved ICP $<20 \mathrm{~mm} \mathrm{Hg}$. Due to persistent need for medical interventions for ICP, the parenchymal monitor was replaced with a single-hole MMM bolt/external ventricular drain (Hummingbird Quad, IRRAS). ${ }^{10}$ Repeated ICP elevation episodes into the mid-20-mm $\mathrm{Hg}$ range were treated with hypertonic saline boluses and sedation. Eventually, pentobarbital sedation was introduced because of persistent elevations despite a relatively benign radiographic appearance. Because of this benign appearance and the ability to keep the ICP range in the upper teens to low 20s, surgical intervention was not deemed necessary. On hospital day 3 (Fig. 2), the patient's ICP continued with only moderate spikes $>20 \mathrm{~mm} \mathrm{Hg}$ (Fig. 2A); however, review of MMM data revealed several worsening trends. $\mathrm{PbO}_{2}$, which had consistently been $>20 \mathrm{~mm} \mathrm{Hg}$, began to decrease below this threshold despite ventilator changes (Fig. 2D). Pressure reactivity (PRx; which was being continuously monitored per the Component Neuromonitoring System [Moberg Research]) had been fluctuating around 0 and began trending significantly higher toward 1 over the course of approximately 6 hours (Fig. 2C). During this time, the automated pupillometry results showed slight asymmetry and decreased reactivity (Neurological Pupil index [NPi]) on the left, which essentially became nonreactive (NPi 0) during the night between days 2 and 3 (Fig. 3). The mean arterial pressure (MAP) and CPP were within normal ranges at 70-100 $\mathrm{mm} \mathrm{Hg}$ and $60-70 \mathrm{~mm} \mathrm{Hg}$, respectively (Fig. 2B). Based on these rapidly worsening indicators of impending herniation despite maximal medical management, the decision was made to perform left DC. The left side was chosen due to the predominance of contusions on the left as well as the left-sided pupillary changes. This was done in the usual fashion with a large bone flap ${ }^{5}$ placed in the abdominal subcutaneous tissue. ${ }^{11}$

After the DC, the patient's ICP stayed less than $10 \mathrm{~mm} \mathrm{Hg}$ without need for drainage or other interventions. Remarkably, the PRx returned to normal nearly immediately, $\mathrm{PbO}_{2}$ increased within several hours of surgery, and the left pupil NPi and size returned to normal and symmetric with the right pupil within hours of surgery.

The patient's neurological examination results improved, and he underwent early tracheostomy. The bone flap was replaced uneventfully 3 weeks after DC, and he was discharged 27 days after injury to an inpatient rehabilitation facility with a GCS score of E4VTM6. At a 4month follow-up visit, the patient was awake and oriented but unable to attend to his own affairs, requiring assistance in activities of daily living.

\section{Discussion \\ Observations}

The decision to perform DC in this patient was influenced largely by MMM in addition to standard ICP measurement. We believe that rapidly worsening $\mathrm{PRx}, \mathrm{PbO}_{2}$, and pupillary reactivity were evidence of impending herniation, and all these physiological measures rapidly improved after DC. Such measures represent additional factors that may help case-by-case decision-making urged in this disease. ${ }^{6}$

Management of TBI with MMM remains understudied. There is a need for an effective means to monitor TBI to guide individualized interventions. ${ }^{5}$ There are many different approaches that may all give some insight into damaging brain physiology that may include inadequate perfusion, inadequate $\mathrm{PbO}_{2}$, impaired autoregulation, spreading 

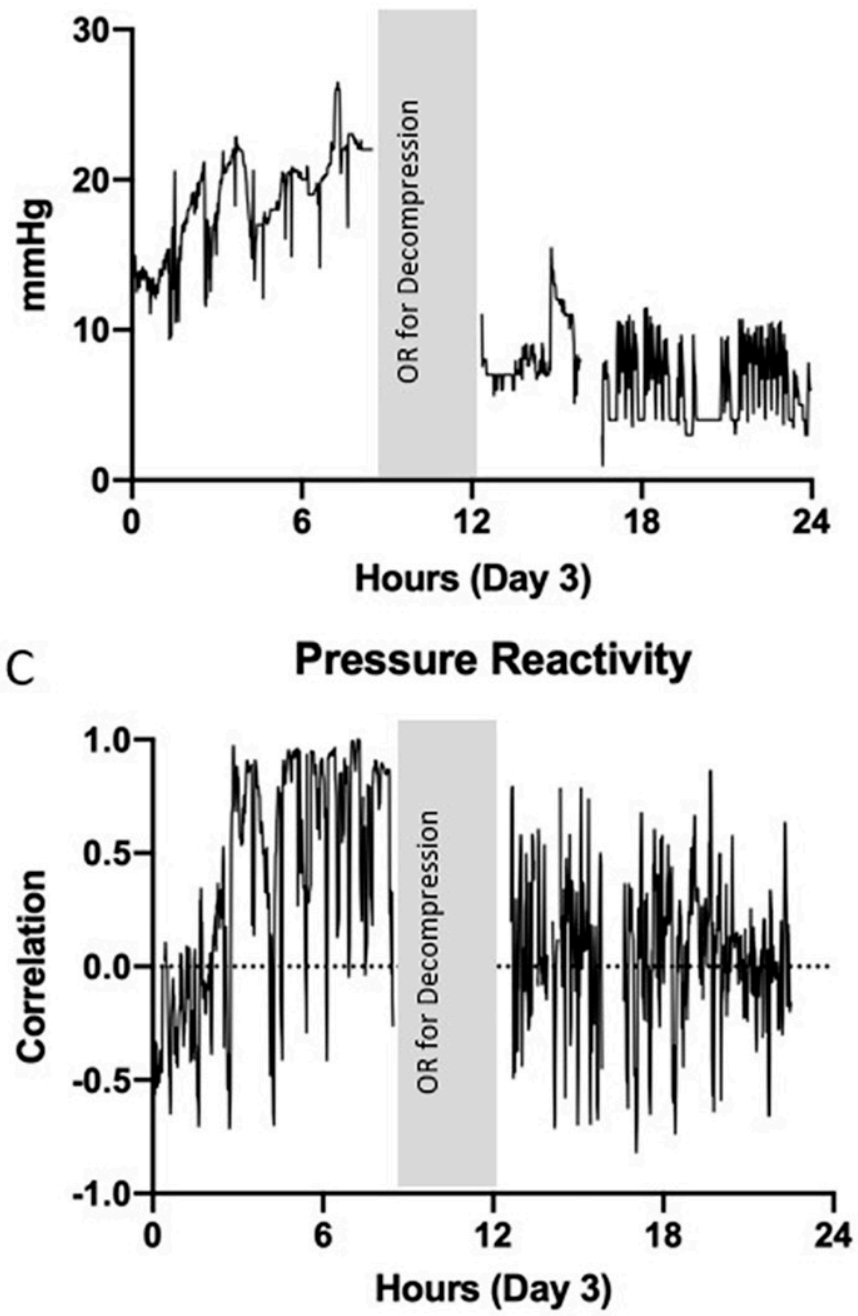

B

MAP and CPP
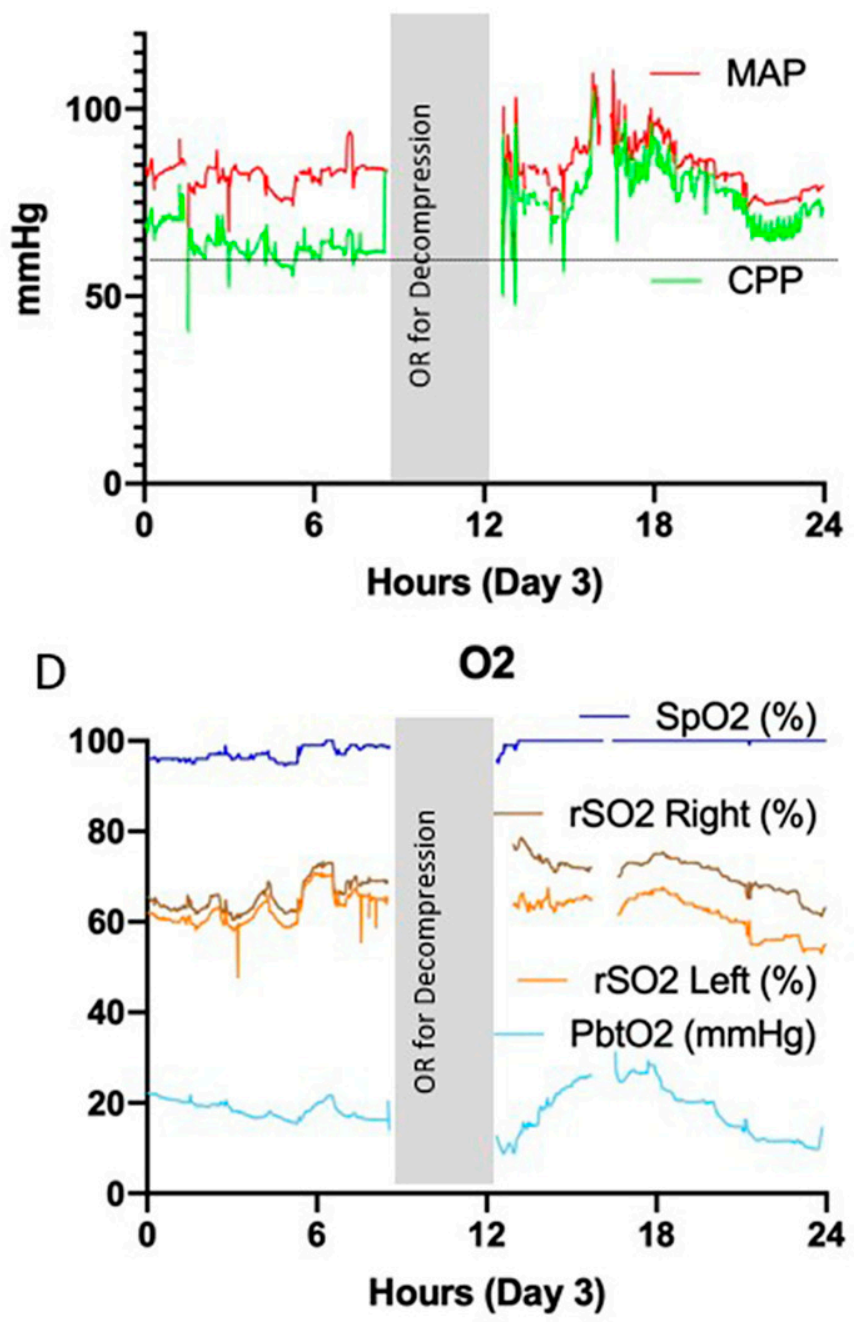

FIG. 2. MMM data on day 3. The vertical gray bar represents the time the patient was in the operating room (OR) for DC. A: Recurring ICP spikes over $20 \mathrm{~mm}$ Hg. B: MAP and CPP within normal ranges, $70-100 \mathrm{~mm} \mathrm{Hg}$ and $60-70 \mathrm{~mm} \mathrm{Hg}$, respectively. Horizontal line represents the threshold level of $60 \mathrm{~mm} \mathrm{Hg}$ for CPP. C: PRx fluctuating less than 0 with a significant increase toward 1 over the course of 6 hours. Dotted line is at PRx of 0 . D: Brain tissue oxygenation (PbtO ${ }_{2}$ ) decreasing below $20 \mathrm{~mm} \mathrm{Hg}$. Note significant improvement in ICP and PRx after decompression. rSO2 = regional oxygen saturation; $\mathrm{SpO} 2=0 \mathrm{xygen}$ saturation.

depolarization and seizures, and impaired metabolism. Although ICP alone is the most commonly used determining factor for medical or surgical intervention, the use of $\mathrm{PbO}_{2}$ (which may be a better estimate of cerebral blood flow $[\mathrm{CBF}]^{12}$ ) has been evaluated. Brain Oxygen Optimization in Severe TBI Phase II (BOOST-2) demonstrated the safety and feasibility of $\mathrm{PbO}_{2}$-directed therapy, ${ }^{9}$ and BOOST-3 is a currently enrolling, double-blind randomized controlled study assessing the efficacy of such an approach.

PRx has been proposed as a surrogate measure of autoregulatory status, which has been associated with outcome and is an attractive target for individualized management based on the optimal CPP. This approach uses the highly time-resolved fluctuations of ICP as a surrogate for CBF, calculating a moving correlation coefficient of ICP and MAP. Retrospective studies have demonstrated worse outcomes in patients with a PRx threshold greater than approximately $0.25 .^{13}$ By measuring PRx across an individual patient's range of spontaneously fluctuating blood pressure or CPP, the level at which the PRx is lowest (blood flow most stable relative to MAP) can be identified and may offer an improved CPP target to individualize care. A clinical trial targeting the feasibility of this approach (COGiTATE) is underway. ${ }^{14}$

The use of automated pupillary measurement and indices is becoming a topic of significant interest, given the noninvasive nature of this approach. Abnormal NPi $(<3)$ in comatose patients was found to be predictive of the need for surgical intervention. ${ }^{7}$ Furthermore, NPi has been associated with both increased ICP and subsequent spikes, supporting the concept that worsening NPi may be a harbinger of herniation and therefore may be used to guide earlier intervention. ${ }^{15}$

\section{Lessons}

This case demonstrates the utility of physiological MMM in addition to ICP when determining management of severe TBI, particularly the need for surgical intervention. Understanding the disturbances in underlying 

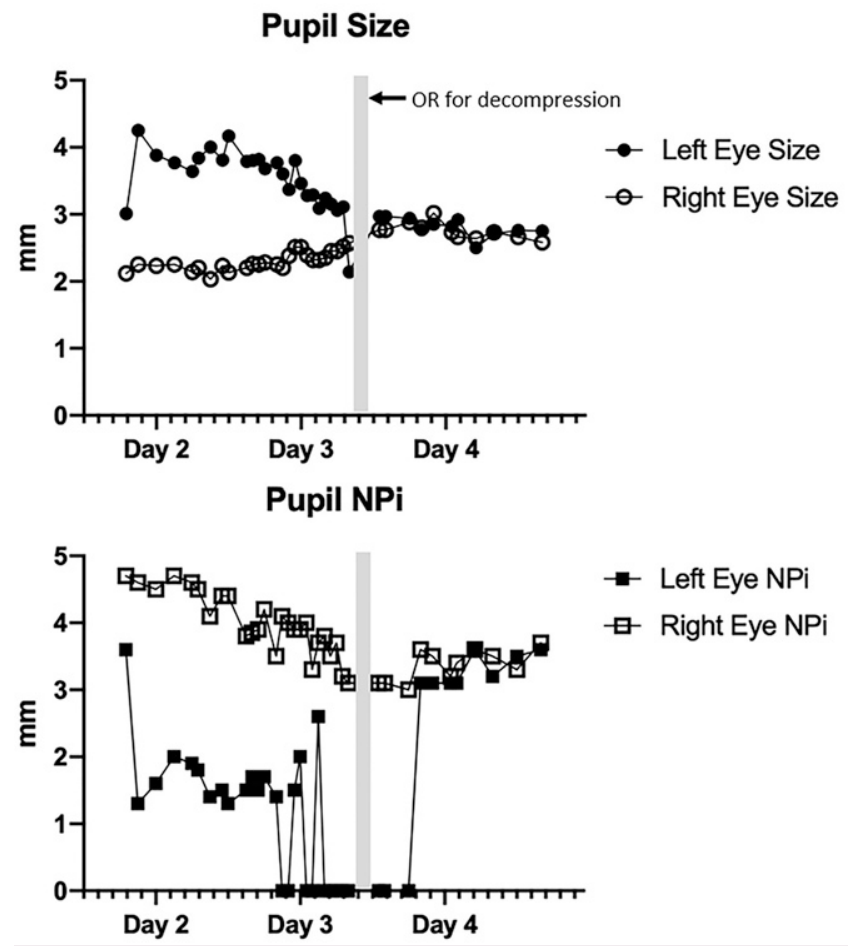

FIG. 3. Automated pupillometry measurements during the first 5 days of admission. Upper: Pupillary size. Note slight asymmetry, which improved after decompression. Lower: Pupillary reactivity (NPi). Lefteye NPi became absent (despite maintained size) between days 2 and 3. Reactivity normalized after decompression. $\mathrm{OR}=$ operating room.

physiology may better allow precision care for these patients, given the complex and conflicting state of the current literature. In addition, such an approach allows better identification of patients with initially more normal physiology whose conditions subsequently deteriorate, which is the optimum group most likely to benefit from aggressive interventions such as DC. ${ }^{6}$

\section{References}

1. Hawryluk GW, Manley GT. Classification of traumatic brain injury: past, present, and future. Handb Clin Neurol. 2015;127:15-21.

2. McHugh GS, Engel DC, Butcher I, et al. Prognostic value of secondary insults in traumatic brain injury: results from the IMPACT study. J Neurotrauma. 2007;24(2):287-293.

3. Shrestha GS, Suarez JI, Hemphill JC III. Precision medicine in neurocritical care. JAMA Neurol. 2018;75(12):1463-1464.

4. Chesnut RM, Petroni G, Rondina $C$. Intracranial-pressure monitoring in traumatic brain injury. N Engl J Med. 2013;368(18):1751-1752.
5. Carney N, Totten AM, O'Reilly C, et al. Guidelines for the Management of Severe Traumatic Brain Injury, Fourth Edition. Neurosurgery. 2017;80(1):6-15.

6. Hawryluk GWJ, Rubiano AM, Totten AM, et al. Guidelines for the Management of Severe Traumatic Brain Injury: 2020 update of the decompressive craniectomy recommendations. Neurosurgery. 2020;87(3):427-434.

7. El Ahmadieh TY, Bedros N, Stutzman SE, et al. Automated pupillometry as a triage and assessment tool in patients with traumatic brain injury. World Neurosurg. 2021;145:e163-e169.

8. Copplestone S, Welbourne J. A narrative review of the clinical application of pressure reactivity indices in the neurocritical care unit. $\mathrm{Br}$ J Neurosurg. 2018;32(1):4-12.

9. Okonkwo DO, Shutter LA, Moore C, et al. Brain oxygen optimization in severe traumatic brain injury phase-II: a phase II randomized trial. Crit Care Med. 2017;45(11):1907-1914.

10. Chohan MO, Akbik OS, Ramos-Canseco J, et al. A novel single twistdrill access device for multimodal intracranial monitoring: a 5-year single-institution experience. Neurosurgery. 2014;10(suppl 3):400-411.

11. Ernst G, Qeadan F, Carlson AP. Subcutaneous bone flap storage after emergency craniectomy: cost-effectiveness and rate of resorption. J Neurosurg. 2018;129(6):1604-1610.

12. Valadka $A B$, Hlatky $R$, Furuya $Y$, Robertson CS. Brain tissue $\mathrm{PO}_{2}$ : correlation with cerebral blood flow. Acta Neurochir Suppl (Wien). 2002:81:299-301.

13. Riemann L, Beqiri E, Younsi A, et al. Predictive and discriminative power of pressure reactivity indices in traumatic brain injury. Neurosurgery. 2020;87(4):655-663.

14. Beqiri $E$, Smielewski $P$, Robba $C$, et al. Feasibility of individualised severe traumatic brain injury management using an automated assessment of optimal cerebral perfusion pressure: the COGiTATE phase II study protocol. BMJ Open. 2019;9(9):e030727.

15. Chen JW, Gombart ZJ, Rogers S, et al. Pupillary reactivity as an early indicator of increased intracranial pressure: the introduction of the Neurological Pupil index. Surg Neurol Int. 2011;2:82.

\section{Disclosures}

The authors report no conflict of interest concerning the materials or methods used in this study or the findings specified in this paper.

\section{Author Contributions}

Conception and design: Carlson, Robinson. Acquisition of data: Carlson, Robinson, Shin. Analysis and interpretation of data: Carlson, Robinson. Drafting the article: Carlson, Robinson, Cole. Critically revising the article: Carlson, Robinson, Alunday, Cole, Torbey. Reviewed submitted version of manuscript: Carlson, Robinson, Shin, Alunday, Torbey. Approved the final version of the manuscript on behalf of all authors: Carlson. Statistical analysis: Carlson. Administrative/ technical/material support: Robinson.

\section{Correspondence}

Andrew P. Carlson: University of New Mexico School of Medicine, Albuquerque, NM. andrewcarlson@salud.unm.edu. 